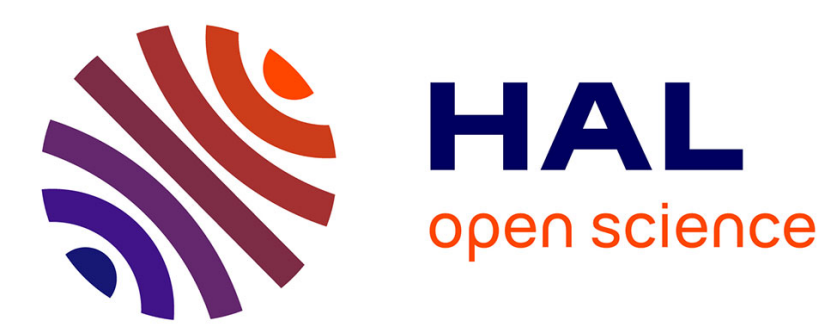

\title{
Computing the codimension of the singularity at the origin for delay systems in the regular case: A vandermonde-based approach
}

Islam Boussaada, Dina Irofti, Silviu-Iulian Niculescu

\section{- To cite this version:}

Islam Boussaada, Dina Irofti, Silviu-Iulian Niculescu. Computing the codimension of the singularity at the origin for delay systems in the regular case: A vandermonde-based approach. European Control Conference (ECC), 2014, Jun 2014, Strasbourg, France. 10.1109/ECC.2014.6862469 . hal-01123598

\author{
HAL Id: hal-01123598 \\ https://hal.science/hal-01123598
}

Submitted on 6 Jul 2020

HAL is a multi-disciplinary open access archive for the deposit and dissemination of scientific research documents, whether they are published or not. The documents may come from teaching and research institutions in France or abroad, or from public or private research centers.
L'archive ouverte pluridisciplinaire HAL, est destinée au dépôt et à la diffusion de documents scientifiques de niveau recherche, publiés ou non, émanant des établissements d'enseignement et de recherche français ou étrangers, des laboratoires publics ou privés. 


\title{
Computing the Codimension of the Singularity at the Origin for Delay Systems in The Regular Case: A Vandermonde-Based Approach
}

\author{
Islam Boussaada, Dina-Alina Irofti and Silviu-Iulian Niculescu
}

\begin{abstract}
The analysis of time-delay systems mainly relies on the identification and the understanding of the spectral values bifurcations when crossing the imaginary axis. One of the most important type of such singularities is when the zero spectral value is multiple. The simplest case in such a configuration is characterized by an algebraic multiplicity two and a geometric multiplicity one known as Bogdanov-Takens singularity. Moreover, in some circumstances the codimension of the zero spectral value exceeds the dimension of the delay-free system of differential equations. To the best of the authors' knowledge, the bound of such a multiplicity was not deeply investigated in the literature. This paper provides an answer to this question for time-delay systems with linear part characterized in the Laplace domain by a quasipolynomial function with non sparse polynomials and without coupling delays.
\end{abstract}

Index Terms - Zero singularity, Eigenvalues multiplicity, Time-delay systems, Center Manifold.

\section{INTRODUCTION}

Generally speaking, an $n$-dimensional linear system of ordinary differential equations $\dot{x}=A x$, where $x \in \mathbb{R}^{n}$ and $A \in \mathcal{M}_{n}(\mathbb{R})$ admits $n$ spectral values that are the $n$ eigenvalues of the matrix $A$. Thus, the codimension of a given spectral value can be at most the dimension of the state space. Likewise, this can be seen from the associated characteristic equation which is a polynomial of degree $n$ in the Laplace variable and has at most $n$ complex roots.

However, when dealing with time-delay systems the situation is different. Consider the following infinite-dimensional system with $N$ constant delays:

$$
\dot{x}=\sum_{i=0}^{N} A_{i} x\left(t-\tau_{i}\right)
$$

where $x=\left(x_{1}, \ldots, x_{n}\right)$ denotes the state-vector, under appropriate initial conditions belonging to the Banach space of continous functions $\mathcal{C}\left(\left[-\tau_{N}, 0\right], \mathbb{R}^{n}\right)$. Here $\tau_{i}, i=1 \ldots N$ are strictly increasing positive constant delays and $\tau_{0}=0$, the matrices $A_{i} \in \mathcal{M}_{n}(\mathbb{R})$ for $i=0 \ldots N$, then the associated characteristic equation is a transcendental equation in the Laplace variable $\lambda$ in which appears exponential terms induced by delays. Under some restrictions on the matrices $A_{i}$, the system has a characteristic function $\Delta: \mathbb{C} \times \mathbb{R}^{N}+$

I. Boussaada is with IPSA \& Laboratoire des signaux et systèmes, (L2S, UMR CNRS 8506), Supélec, 3 rue Joliot-Curie, 91192 Gif-sur-Yvette cedex, France is lam.boussaadadlss. supelec.fr

D-A. Irofti is with Laboratoire des signaux et systèmes, (L2S, UMR CNRS 8506), Supélec, 3 rue Joliot-Curie, 91192 Gif-sur-Yvette cedex, France dina.iroftialss.supelec.fr

S-I. Niculescu is with Laboratoire des signaux et systèmes, (L2S, UMR CNRS 8506), Supélec, 3 rue Joliot-Curie, 91192 Gif-sur-Yvette cedex, France Silviu.Niculescullss.supelec.fr
$\mathbb{C}$ of the form:

$$
\Delta(\lambda, \tau)=P_{0}(\lambda)+\sum_{i=1}^{N} P_{i}(\lambda) e^{-\tau_{i} \lambda}
$$

or shortly $\Delta(\lambda)$. Without any loss of generality, assume that the polynomial $P_{0}$ is monic polynomial of degree $n$ in $\lambda$ and the polynomials $P_{i}$ are such that $\operatorname{deg}\left(\mathrm{P}_{\mathrm{i}}\right) \leq(\mathrm{n}-1), \forall 1 \leq \mathrm{i} \leq$ $\mathrm{N}$. Note that the integer $n$ is nothing else than dimension of the delay-free differential equations associated with system (1). One can prove that the quasipolynomial function (2) admits an infinite number of zeros, see [1], [2]. The study of zeros of (2) plays a crucial role in the analysis of asymptotic stability of the zero solution of system (1). Indeed, the zero solution is asymptotically stable if all the zeros of (2) are in the open left half complex plane [3]. Accordingly to this definition, the parameter space which is spanned by the coefficients of the polynomials $P_{i}$, can be split into stability and instability domains (Nothing else that the so-called Ddecomposition, see [3] and references therein). These two domains are separated by a boundary corresponding to a spectra consisting in roots with zero real parts and roots with negative real part. Moreover, under appropriate algebraic restrictions, a given root associated to that boundary can have high multiplicity. In this work we are concerned by the codimension of the zero spectral value. The typical example for non-simple zero spectral value is the Bogdanov-Takens singularity which is characterized by an algebraic multiplicity two and a geometric multiplicity one. Cases with higher order multiplicities of the zero spectral value are known to us as generalized Bogdanov-Takens singularities. Those types of configurations are not necessarily synthetically and are involved in concrete applications. Indeed, the BogdanovTakens singularity is identified in [4] where the case of two coupled scalar delay equations modeling a physiological control problem is studied. In [5], this type of singularity is also encountered in the study of coupled axial-torsional vibrations of an oilwell rotary drilling system. Moreover, the paper [6] is dedicated to this type of singularities, codimensions two and three are studied and the associated center manifold are explicitly computed. Commonly, the time-delay induces desynchronizing and/or destabilizing effect on the dynamics. However, new theoretical developments in control of finitedimensional dynamical systems suggest the use of delays in the control laws for stabilization purposes. For instance, the papers [7], [8] are concerned by the stabilization of the inverted pendulum by delayed control laws and furnish concrete situations where the codimension of the zero spectral value exceeds the number of the coupled scalar equations 
modeling the inverted pendulum on cart. In [7], the authors prove that delayed proportional-derivative (PD) controller stabilize the inverted pendulum by identifying a codimension three singularity for a system of two coupled delayed equations. In [8], the same singularity is characterized by using a particular delay block configuration. It is shown that two delay blocks offset a PD delayed controller. Although the algebraic structure of the multiplicity problem makes the finite aspect of such a codimension evident, to the best of the authors' knowledge, the question: on the upper bound of the codimension of the zero spectral value did not receive a complete characterization.

In this paper, we investigate this type of singularity and give an answer to the question above. This work is motivated by the fact that the knowledge of such information is crucial when dealing with a nonlinear analysis and the center manifold computations are involved. Indeed, when the zero spectral value is the only eigenvalue with zero real part, then the center manifold dimension is none other than the codimension of the generalized Bogdanov-Takens singularity [9], [10], [11], [12].

The effective method elaborated in this paper emphasizes the connexions between the codimension problem and Generalized confluent Vandermonde matrices. To the best of the author knowledge, the first time the Vandermonde matrix appears in a control problem is reported in [13], where the controllability of a finite dimensional dynamical system is guaranteed by the invertibility of such a matrix, see [13, p. 121]. Next, in the context of time-delay systems, the use of Vandermonde matrix properties was proposed by [14], [3] when controlling one chain of integrators by delay blocks. Here we further exploit the algebraic properties of such matrices into a different context.

The remaining paper is organized as follows. Section 2 includes a motivating example. Next, the main results are proposed in Section 3 and 4. Various illustrative examples are presented in Section 5. Finally, some concluding remarks end the paper.

\section{A motivating EXAMPle: InVERTed Pendulum}

As a classical mechanical example, we consider a system of two coupled equations with two delays modeling a friction free inverted pendulum on cart. The adopted model is studied in [15], [7], [16], [8] for which we keep the same notations. In the dimensionless form, the dynamics of the inverted

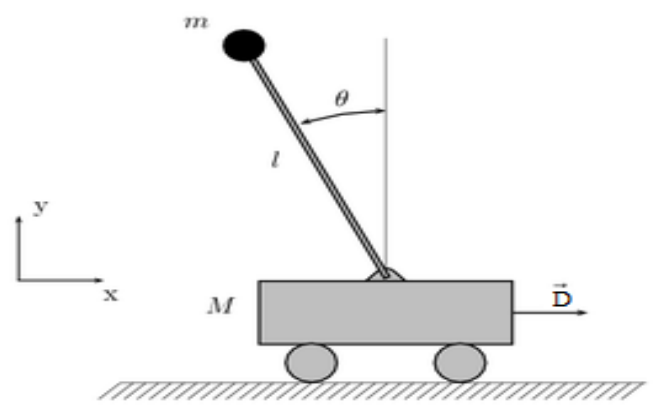

Fig. 1. Inverted Pendulum on a cart pendulum on a cart in figure 1 is governed by the following second-order differential equation:

$$
\left(1-\frac{3 \epsilon}{4} \cos ^{2}(\theta)\right) \ddot{\theta}+\frac{3 \epsilon}{8} \dot{\theta}^{2} \sin (2 \theta)-\sin (\theta)+D \cos (\theta)=0,
$$

where $\epsilon=m /(m+M), M$ the mass of the cart and $m$ the mass of the pendulum and $D$ represents the control law that is the horizontal driving force. A generalized BogdanovTakens singularity with codimension three is identified in [7] by using $D=a \theta(t-\tau)+b \dot{\theta}(t-\tau)$. Motivated by the technological constraints, it is suggested in [8] to avoid the use of the derivative gain that requires the estimation of the angular velocity that can induce harmful errors for realtime simulations and propose a multi-delayed-proportional controller $D=\gamma_{1} \theta\left(t-\tau_{1}\right)+\gamma_{2} \theta\left(t-\tau_{2}\right)$, this choice is argued by the accessibility of the delayed state by simpler sensor. When we set $\epsilon=\frac{3}{4}$, the associated quasipolynomial characteristic function $\Delta$ becomes:

$$
\Delta(\lambda)=\lambda^{2}-\frac{16}{7}+\frac{16 \gamma_{1}}{7} \mathrm{e}^{-\lambda \tau_{1}}+\frac{16 \gamma_{2}}{7} \mathrm{e}^{-\lambda \tau_{2}}
$$

A zero singularity with codimension three is identified in [8]. Moreover, it is shown that the upper bound of the codimension for the zero singularity for (3) is three and this configuration is obtained when the gains and delays satisfy simultaneously:

$$
\gamma_{1}=-\frac{7}{-7+8 \tau_{1}^{2}}, \gamma_{2}=\frac{8 \tau_{1}^{2}}{-7+8 \tau_{1}^{2}}, \tau_{2}=\frac{7}{8 \tau_{1}} .
$$

\section{Main Results}

Let us first recall some useful definitions:

A polynomial $P$ of degree $n$ is said to be sparse when $P(x)=\sum_{k=0}^{n} a_{k} x^{k}$ and $\prod_{k=0}^{n-1} a_{k}=0$, see for instance [17].

In the sequel, by regular qusipolynomial we understand the situation when the polynomials $P_{i}$ in (2) for $i=1, \ldots, N$ are not sparse i.e. $\forall i=1, \ldots, N$ and $\forall k=0, \ldots, \operatorname{deg}\left(P_{i}\right)$ we assume that $a_{i, k} \neq 0$.

The main result can be summarized as follows:

Proposition 1: The codimension of zero singularity of the regular characteristic quasipolynomial function $\Delta$ given by (2) cannot be larger than $N\left(n_{0}+1\right)+n$, where $N$ is the number of the nonzero distinct delays and $n$ is the degree of $P_{0}$ and $n_{0}$ is the upper degree of the polynomial family $P_{i}$, for $1 \leq i \leq N$.

The proof of can be found in appendix.

Remark 2: Please notice that several other notions can be encountered in the literature describing sparsity. Among others we mention the lacunary polynomials [18].

Remark 3: In the light of the result of Proposition III, we are able to establish the codimension's upper bound of the zero singularity of the characteristic quasipolynomial function $\Delta$ when all its parameters are left free. Indeed, in such a case, it is assumed that $n_{0}=\max _{1 \leq i \leq N} \operatorname{deg}\left(P_{i}\right)$ is exactly $n-1$ and thus we claim:

The codimension of zero singularity of the characteristic quasipolynomial function $\Delta$ given by (2) cannot be larger than $(N+1) n$, where $N$ is the number of the nonzero distinct 
delays and $n$ is the degree of $P_{0}$. This bound is the same as the one from the Polya-Szegö result in [19] which gives a bound for the number of quasipolynomial roots that are contained in a given horizontal strip. Note that the proof of Polya-Szegö result is based on Rouché theorem, however, in this paper, we established a constructive approach.

Remark 4: It is important to emphasize that the codimension's upper bound exceeds the number of free parameters involved in the quasipolynomial function $\Delta$. Indeed, the number of free parameters is $N(n+1)+n$ which is greater than $(N+1) n$.

\section{LINK WITH VANDERMONDE MATRICES}

In the sequel, by generalized confluent Vandermonde matrix $W$ we associate to a given positive integer $s \geq 0$ the square matrix defined by:

$$
\begin{gathered}
W=\left[W_{1} W_{2} \ldots W_{M}\right] \in \mathcal{M}_{\delta}(\mathbb{R}), \\
\text { where } W_{i}=\left[f\left(x_{i}\right) f^{(1)}\left(x_{i}\right) \ldots f^{\left(d_{i}-1\right)}\left(x_{i}\right)\right]
\end{gathered}
$$

such that $\sum_{i=1}^{M} d_{i}=\delta$ and

$$
f\left(x_{i}\right)=\left[x_{i}^{s} \ldots x_{i}^{\delta+s-1}\right]^{T}, \quad \text { for } \quad 1 \leq i \leq M .
$$

When $s=0$, the matrix $W$ is said a confluent Vandermonde matrix and thus $f\left(x_{i}\right)=\left[1 x_{i} \ldots x_{i}^{\delta-1}\right]^{T}$. If in addition, $d_{i}=$ 1 for $i=1 \ldots N$ then $W$ is simply said a Vandermonde matrix and in this case $M=\delta$ since $W$ is assumed to be a square matrix.

Let $\xi$ stands for the vector composed from $x_{i}$ counting their repetition $d_{i}$ through columns of $W$, that is

$$
\xi=(\underbrace{x_{1}, \ldots, x_{1}}_{d_{1}}, \ldots, \underbrace{x_{M}, \ldots, x_{M}}_{d_{M}}) .
$$

For instance one has $\xi_{1}=x_{1}$ and $\xi_{d_{1}+d_{2}+1}=$ $\xi_{d_{1}+d_{2}+d_{3}}=x_{3}$. In the light of the above notations and under the setting $d_{0}=0$, without any loss of generality: $\xi_{k}=$ $\xi_{d_{0}+\ldots+d_{r}+\alpha}=\xi_{\sum_{l=0}^{\varrho(k)-1} d_{l}+\varkappa(k)}$, where $0 \leq r \leq M-1$ and $\alpha \leq d_{r+1}$, here $\varrho(\bar{k})$ denotes the index of component of $x$ associated with $\xi_{k}$, that is $x_{\varrho(k)}=\xi_{k}$ and by $\varkappa(k)$ the order of $\xi_{k}$ in the sequence of $\xi$ composed only by $x_{\varrho(k)}$. Obviously, $\varrho(k)=r+1$ and $\varkappa(k)=\alpha$.

The following theorem provides the $L U-$ factorization of the matrix $W$.

Theorem 5: Given the generalized confluent Vandermonde matrix (4)-(6), the unique LU-factorization with unitary diagonal elements $L_{i, i}=1$ is given by the formulae:

$$
\left\{\begin{aligned}
L_{i, 1}= & x_{1}^{i-1} \text { for } 1 \leq i \leq \delta, \\
U_{1, l}= & W_{1, l} \text { for } 1 \leq l \leq \delta, \\
L_{i, l}= & L_{i-1, l-1}+L_{i-1, l} \xi_{l} \text { for } 2 \leq l \leq i, \\
U_{i, l}= & (\varkappa(l)-1) U_{i-1, l-1} \\
& +U_{i-1, l}\left(x_{\varrho(l)}-\xi_{i-1}\right) \quad \text { for } 2 \leq i \leq l .
\end{aligned}\right.
$$

The proof of the above theorem is given in [20].

\section{Illustration OF CODIMENSION $N\left(n_{0}+1\right)+n$ AND CONTROLling Generalized Bogdanov-TAKENS \\ SINGULARITY}

A natural consequence of Proposition 1 is to explore the situation when the codimension is equal to $N\left(n_{0}+1\right)+n$ that is the codimension of zero singularity reaches its upper bound and, particularly, when the codimension is equal $(N+$ 1) $n$ we say that the codimension of zero singularity reaches its optimal value. Examples below complete the introductory motivating example.

A. Two coupled scalar equations with two delays: the codimension's upper bound

Consider the system of two coupled scalar equations with two time-delays:

$$
\dot{x}=\sum_{k=0}^{2} A_{k} x\left(t-\tau_{k}\right)
$$

where the real valued matrices $A_{k}$ are such that:

$$
A_{0}=\left(\begin{array}{cc}
0 & b_{1,2} \\
b_{2,1} & b_{2,2}
\end{array}\right), A_{1}=\left(\begin{array}{cc}
0 & 0 \\
c_{2,1} & c_{2,2}
\end{array}\right), A_{2}=\left(\begin{array}{cc}
0 & 0 \\
d_{2,1} & d_{2,2}
\end{array}\right) .
$$

The associated characteristic matrix is given by

$$
C(\lambda)=\left(\begin{array}{cc}
\lambda & -b_{1,2} \\
C_{2,1} & C_{2,2}
\end{array}\right)
$$

where $C_{2,1}=-b_{2,1}-\mathrm{e}^{-\lambda \tau_{1}} c_{2,1}-\mathrm{e}^{-\lambda \tau_{2}} d_{2,1}, \quad C_{2,2}=$ $\lambda-b_{2,2}-\mathrm{e}^{-\lambda \tau_{1}} c_{2,2}-\mathrm{e}^{-\lambda \tau_{2}} d_{2,2}$ such that the associated characteristic transcendental function $\Delta$ becomes:

$$
\begin{aligned}
\Delta(\lambda, \tau) & =\lambda^{2}-\lambda b_{2,2}-b_{1,2} b_{2,1}+\left(-b_{1,2} c_{2,1}-\lambda c_{2,2}\right) \mathrm{e}^{-\lambda \tau_{1}} \\
& +\left(-b_{1,2} d_{2,1}-\lambda d_{2,2}\right) \mathrm{e}^{-\lambda \tau_{2}} .
\end{aligned}
$$

To identify the parameter values characterizing a zero singularity of codimension 6 we have to compute the variety $V$ associated with the ideal $I$ such that:

$$
\begin{aligned}
& I=<b_{1,2} c_{2,1}+b_{1,2} d_{2,1}+b_{1,2} b_{2,1}, \\
& c_{2,2}-b_{1,2} c_{2,1} \tau_{1}+d_{2,2}-b_{1,2} d_{2,1} \tau_{2}+b_{2,2}, \\
& \quad-2 \tau_{1} c_{2,2}+b_{1,2} c_{2,1} \tau_{1}{ }^{2}-2 \tau_{2} d_{2,2}+b_{1,2} d_{2,1} \tau_{2}{ }^{2}-2, \\
& 3 \tau_{1}{ }^{2} c_{2,2}-b_{1,2} c_{2,1} \tau_{1}{ }^{3}+3 \tau_{2}{ }^{2} d_{2,2}-b_{1,2} d_{2,1} \tau_{2}{ }^{3}, \\
& \quad-4 \tau_{1}{ }^{3} c_{2,2}+b_{1,2} c_{2,1} \tau_{1}{ }^{4}-4 \tau_{2}{ }^{3} d_{2,2}+b_{1,2} d_{2,1} \tau_{2}{ }^{4} \\
& 5 \tau_{1}{ }^{4} c_{2,2}-b_{1,2} c_{2,1} \tau_{1}{ }^{5}+5 \tau_{2}{ }^{4} d_{2,2}-b_{1,2} d_{2,1} \tau_{2}{ }^{5}>.
\end{aligned}
$$

Proposition 6: The system (7)-(8) admits a zero singularity of codimension 6 if and only if $b_{1,2}^{2}+d_{2,1}^{2} \neq 0$ and one of the following assertions hold:

1) $d_{2,1}=0$ and

$$
\begin{aligned}
& b_{2,1}=-\frac{324}{25 b_{1,2} \tau_{2}^{2}}, b_{2,2}=\frac{32}{5 \tau_{2}}, c_{2,1}=\frac{324}{25 b_{1,2} \tau_{2}^{2}}, \\
& c_{2,2}=\frac{27}{10 \tau_{2}}, d_{2,2}=\frac{25}{2 \tau_{2}}, \tau_{1}=\frac{5 \tau_{2}}{3} .
\end{aligned}
$$

2) $d_{2,1} \neq 0$ and

$$
\begin{aligned}
& b_{1,2}=2 \frac{\tau_{1}^{2}\left(3 \tau_{1}-5 \tau_{2}\right)}{\tau_{2}^{2}\left(\tau_{1}-\tau_{2}\right)^{3} d_{2,1}}, d_{2,2}=2 \frac{\tau_{1}^{2}}{\left(\tau_{1}-\tau_{2}\right)^{2} \tau_{2}}, \\
& c_{2,1}=\frac{d_{2,1}\left(5 \tau_{1}-3 \tau_{2}\right) \tau_{2}^{4}}{\tau_{1}^{4}\left(3 \tau_{1}-5 \tau_{2}\right)}, c_{2,2}=2 \frac{\tau_{2}{ }^{2}}{\tau_{1}\left(\tau_{1}-\tau_{2}\right)^{2}} \\
& b_{2,1}=-\frac{d_{2,1}\left(5 \tau_{1} \tau_{2}^{4}-3 \tau_{2}{ }^{5}+3 \tau_{1}^{5}-5 \tau_{2} \tau_{1}^{4}\right)}{\tau_{1}^{4}\left(3 \tau_{1}-5 \tau_{2}\right)} \\
& b_{2,2}=4 \frac{\tau_{2}+\tau_{1}}{\tau_{2} \tau_{1}} .
\end{aligned}
$$


Moreover, for these two cases, the zero singularity belong to the class of generalized Bogdanov-Takens type (geometric multiplicity one).

Proof: Let us consider first the case where $b_{1,2}=$ $d_{2,1}=0$. Then, the ideal $I$ becomes:

$$
\begin{aligned}
& I=<c_{2,2}+d_{2,2}+b_{2,2},-2-2 \tau_{1} c_{2,2}-2 \tau_{2} d_{2,2}, \\
& 3 \tau_{1}{ }^{2} c_{2,2}+3 \tau_{2}{ }^{2} d_{2,2},-4 \tau_{1}^{3} c_{2,2}-4 \tau_{2}{ }^{3} d_{2,2}, \\
& 5 \tau_{1}{ }^{4} c_{2,2}+5 \tau_{2}{ }^{4} d_{2,2}>,
\end{aligned}
$$

which has an empty variety. Otherwise, $b_{1,2}^{2}+d_{2,1}^{2} \neq 0$ and the ideal $I$ consists on linear equations (when $\tau$ is considered as a parameter). The vanishing of such equations allows to a linear system $M_{2} a=0$, where $M_{2} \in \mathcal{M}_{n}(\mathbb{R}[\tau])$, leading to the two complementary sub-varieties given in the Proposition statement. Moreover, when $d_{2,1}=0$, the characteristic matrix is written $C(\lambda)=$

$$
\left(\begin{array}{cc}
\lambda & -b_{1,2} \\
\frac{324}{25} \frac{1-\mathrm{e}^{-\frac{5}{3} \lambda \tau_{2}}}{b_{1,2} \tau_{2}{ }^{2}} & \lambda-\frac{32}{5} \tau_{2}{ }^{-1}-\frac{27}{10} \frac{\mathrm{e}^{-\frac{5}{3} \lambda \tau_{2}}}{\tau_{2}}-\frac{25}{2} \frac{\mathrm{e}^{-\lambda \tau_{2}}}{\tau_{2}}
\end{array}\right)
$$
and the zero singularity has only one eigenvector $(1,0)^{T}$ and a basis $\Phi_{1}$ for the associated generalized eigenspace $\Phi_{1}(\theta)=$

$$
\left(\begin{array}{cccccc}
1 & \theta & \frac{\theta^{2}}{2}+3 & \frac{\theta^{3}}{6}+3 \theta & \frac{\theta^{4}}{24}+\frac{3 \theta^{2}}{2} & \frac{\theta^{5}}{120}+\frac{\theta^{3}}{2}+1 \\
0 & 1 & \theta & \frac{\theta^{2}}{2}+3 & \frac{\theta^{3}}{6}+3 \theta & \frac{\theta^{4}}{24}+\frac{3 \theta^{2}}{2}
\end{array}\right),
$$

satisfying $\Phi_{1}^{\prime}(\theta)=B \Phi_{1}(\theta)$ where $B$ is the superdiagonal shift matrix and the zero singularity is of the generalized Bogdanov-Takens type. The second case, when $d_{2,1} \neq 0$ the basis $\Phi_{2}$ of the generalized eigenspace associated with the codemension six zero singularity is given by $\Phi_{2}(\theta)^{T}=$ $\left[\phi_{k i}\right]$, with $\phi_{11}=1, \phi_{21}=2+\theta, \phi_{31}=3+2 \theta+\frac{\theta^{2}}{2}$, $\phi_{41}=2+3 \theta+\theta^{2}+\frac{\theta^{3}}{6}, \phi_{51}=1+2 \theta+\frac{3 \theta^{2}}{2}+\frac{\theta^{3}}{3}+\frac{\theta^{4}}{24}$, $\phi_{61}=1+\theta+\theta^{2}+\frac{\theta^{3}}{2}+\frac{\theta^{4}}{12}+\frac{\theta^{5}}{120}, \phi_{12}=0, \phi_{22}=\frac{\alpha}{2}, \phi_{32}=$ $\alpha+\frac{\theta \alpha}{2}, \phi_{42}=\frac{3 \alpha}{2}+\theta \alpha+\frac{\theta^{2} \alpha}{4}, \phi_{52}=\alpha+\frac{3 \theta \alpha}{2}+\frac{\theta^{2} \alpha}{2}+\frac{\theta^{3} \alpha}{12}$, $\phi_{62}=\frac{\alpha}{2}+\theta \alpha+\frac{3 \theta^{2} \alpha}{4}+\frac{\theta^{3} \alpha}{6}+\frac{\theta^{4} \alpha}{48}$, where $\alpha=\frac{\tau_{2}{ }^{2}\left(\tau_{1}-\tau_{2}\right)^{3} d_{2,1}}{\tau_{1}{ }^{2}\left(3 \tau_{1}-5 \tau_{2}\right)}$ also satisfying $\Phi_{2}^{\prime}(\theta)=B \Phi_{2}(\theta)$.

\section{B. Double inverted pendulum with delayed feedback}

We consider a double inverted pendulum described in figure 2, where $\theta_{1}, \theta_{2}$ are the angular position of the lower and upper pendulum with respect to the vertical axis, and $\mathrm{r}$ is the position of the cart with respect to a reference point.

The state-space representation of the system is (see for more details [21] and [22]):

$$
\dot{x}(t)=A_{0} x(t)+A_{1} x\left(t-\tau_{1}\right)+A_{2} x\left(t-\tau_{2}\right),
$$

where $x=\left(\begin{array}{ll}\theta & \dot{\theta}\end{array}\right)^{T} \in \mathbb{R}^{6}, 1 \leq i \leq 2$,

$A_{0}=\left(\begin{array}{cccc}0 & 0_{3} & & \mathrm{I}_{3} \\ 0 & -\frac{441}{46} & \frac{147}{230} & \\ 0 & \frac{2499}{23} & -\frac{3087}{115} & 0_{3} \\ 0-\frac{3087}{23} & \frac{7791}{115} & \end{array}\right) ; A_{i}=\left(\begin{array}{cccc}0 & \frac{14 \gamma_{i 1}}{23} & \frac{14 \gamma_{i 2}}{23} & 0_{3} \\ 0-\frac{36 \gamma_{i 1}}{23} & -\frac{3 \gamma_{i 2}}{23} & 0_{3} \\ 0 & \frac{12 \gamma_{i 1}}{23} & \frac{12 \gamma_{i 2}}{23} & \end{array}\right)$

$\gamma_{i h}$ above are the gains of the command $\mathrm{u}$ of the form

$u=\gamma_{11} \theta_{1}\left(t-\tau_{1}\right)+\gamma_{12} \theta_{2}\left(t-\tau_{1}\right)+\gamma_{21} \theta_{1}\left(t-\tau_{2}\right)+\gamma_{22} \theta_{2}\left(t-\tau_{1}\right)$,

and $\tau_{1}$ and $\tau_{2}$, satisfying $0<\tau_{1}<\tau_{2}$ are the delays.

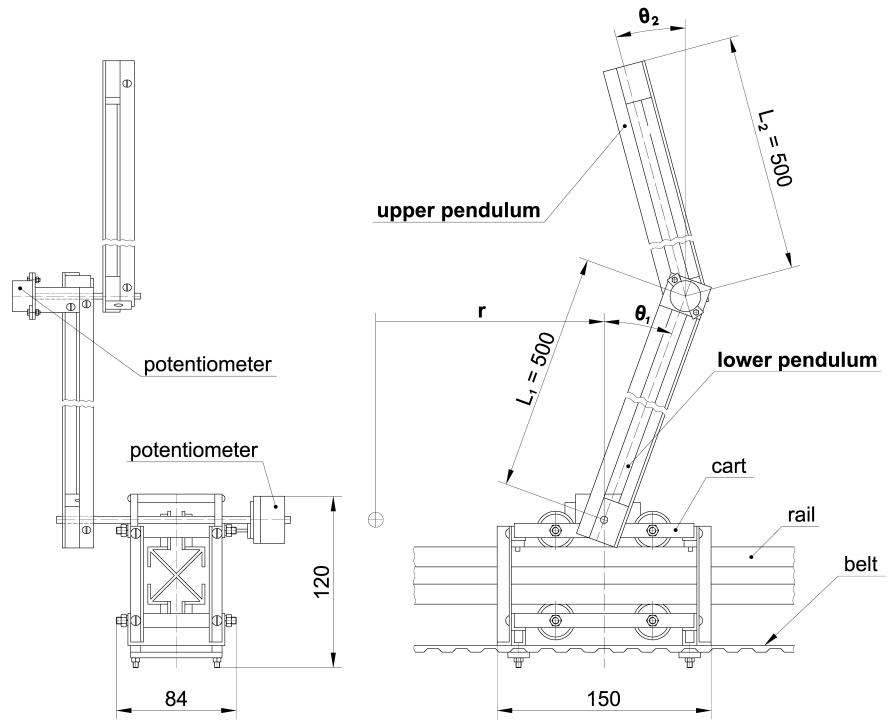

Fig. 2. Double Inverted Pendulum

The characteristic equation of (9) is $\operatorname{det} \Delta(\lambda, \tau)=0$, where the characteristic transcendental function $\Delta(\lambda, \tau)=\lambda \mathrm{I}_{6}-$ $A_{0}-A_{1} \mathrm{e}^{-\lambda \tau_{1}}-A_{2} \mathrm{e}^{-\lambda \tau_{2}}$ can be written as:

$$
\begin{gathered}
\Delta(\lambda, \tau)=P_{0}(\lambda)+\sum_{i=1}^{2} P_{i}(\lambda) e^{-\lambda \tau_{i}}, \text { where } \\
P_{0}(\lambda)=\lambda^{6}-\frac{882}{5} \lambda^{4}+\frac{86436}{23} \lambda^{2}, P_{i}(\lambda)= \\
=-\frac{12}{23} \lambda^{4} \gamma_{i 2}+\frac{36}{23} \lambda^{4} \gamma_{i 1}-\frac{10584}{115} \gamma_{i 1} \lambda^{2}-\frac{3528}{23} \lambda^{2} \gamma_{i 2} .
\end{gathered}
$$

Proposition 7: Under the effect of the delayed feedback given by (10) the multiplicity of the zero spectral value for system (9) is at most 7.

Proof: It is easy to see that $\Delta(0)=\Delta^{\prime}(0)=0$, since $\lambda^{2}$ is a common factor for all $P_{i}$, with $i \epsilon\{0,1,2\}$. Moreover, if $\gamma_{11}$ has the form (11), with the parameters $\gamma_{12}, \gamma_{21}$ and $\gamma_{22}$ left free,

$$
\gamma_{11}=\frac{245}{6}-\frac{5}{3} \gamma_{22}-\gamma_{21}-\frac{5}{3} \gamma_{12},
$$

then the multiplicity at the origin is at least 3 , since $\Delta^{(2)}(0)=0$. Next, we compute the third derivative of $\Delta(0)$, under the condition (11), in order to check if there is a real set of $\left(\gamma_{11}, \gamma_{12}, \gamma_{21}, \gamma_{22}, \tau_{1}, \tau_{2}\right)$ with $0<\tau_{1}<\tau_{2}$ that satisfies $\Delta^{(3)}(0)=0$. Thus, we find the condition

$$
\gamma_{21}=-\frac{5}{6} \frac{2 \gamma_{22} \tau_{1}-2 \tau_{2} \gamma_{22}-49 \tau_{1}}{\tau_{1}-\tau_{2}}
$$

Now we know that if the system's parameters satisfy (11) and (12), $\gamma_{12}, \gamma_{22}, \tau_{1}$ and $\tau_{2}$ are left free and $0<\tau_{1}<\tau_{2}$, then the multiplicity at the origin is at least 4 (since $\Delta(0)$ and its first three derivatives at origin are zero). Following the same reasoning, we are looking for constraints on $\gamma_{12}$, $\gamma_{22}, \tau_{1}$ and $\tau_{2}$ such that the next derivative of $\Delta$ at the origin vanishes, and we obtain

$$
\gamma_{12}=\frac{2401}{4} \tau_{1} \tau_{2}-\gamma_{22}-\frac{539}{15} .
$$

So, under (11)-(13) the multiplicity at the origin is at least 5. Furthermore, if $\gamma_{22}=\frac{49}{60} \frac{\tau_{1}\left(490 \tau_{1} \tau_{2}-245 \tau_{2}{ }^{2}-44\right)}{\tau_{1}-\tau_{2}}$ hold, the 
multiplicity at the origin is at least 6 , and in addition, for the sixth derivative of $\Delta$ at the origin to vanish, the condition (14) must be also satisfied,

$$
\begin{aligned}
& -\frac{2593080 \tau_{1}^{3} \tau_{2}}{23}+\frac{7779240 \tau_{1}^{2} \tau_{2}^{2}}{23} \\
& -\frac{2593080 \tau_{1} \tau_{2}{ }^{3}}{23}-63504 \tau_{1} \tau_{2}+720=0 .
\end{aligned}
$$

Given that the delays are real positive numbers, the condition (14) restricts the domain in which $\tau_{1}$ and $\tau_{2}$ can take values to $\tau_{1} \epsilon(0,0.55)$ and $\tau_{2} \epsilon(0,0.65)$, as we can see in figure 3. Next, we can easily check that $\Delta^{(7)} \neq 0$, i.e. there is no set of real values for $\left(\gamma_{11}, \gamma_{12}, \gamma_{21}, \gamma_{22}, \tau_{1}, \tau_{2}\right)$ with $0<\tau_{1}<\tau_{2}$, that satisfies $\Delta^{(7)}=0$, so the multiplicity at the origin is 7 .

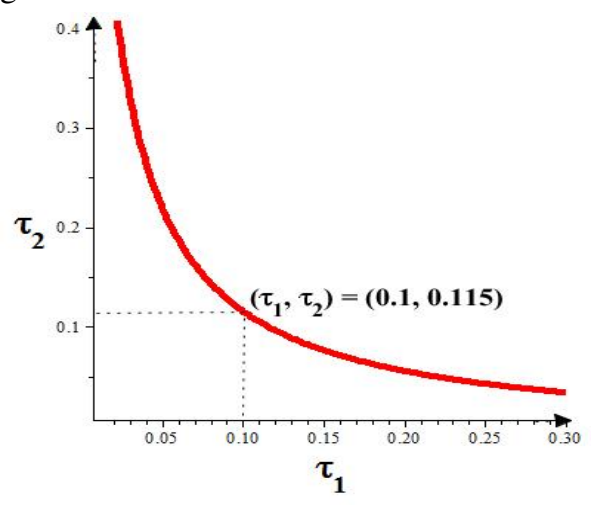

Fig. 3. $\tau_{1}$ and $\tau_{2}$ domain

Remark 8: If we chose a value for $\tau_{1}$ contained in the restricted domain $\left(\tau_{1}=0.1\right.$ for instance), we obtain $\gamma_{11}=$ 709.783, $\gamma_{12}=-245.367, \gamma_{21}=-620.637, \gamma_{22}=216.379$ and $\tau_{2}=0.115$. If we replace those values in the polynomials $P_{0}, P_{1}$ and $P_{2}$, the coefficients obtained verify (A.1) for $1 \leq$ $k \leq 6$ and $1 \leq i \leq 2$. Then, the spectra can be represented like in figure 4 . We notice that we have no spectral values in the right half-plane.

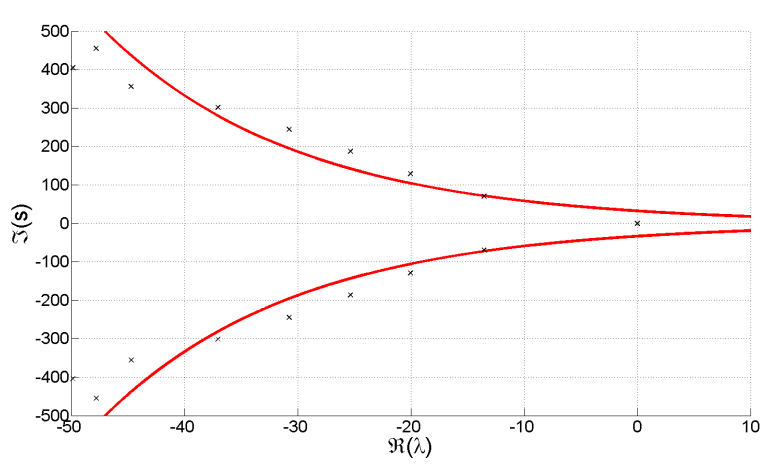

Fig. 4. Spectrum distribution for $\tau_{1}=0.1$ and $\tau_{2}=0.115$

\section{CONCLUSION}

This paper addressed the problem of identifying the maximal dimension of the generalized eigenspace associated with a zero singularity for a class of quasipolynomials. Under the assumption that all the imaginary roots are located at the origin, our result gives the relation between $d$ the maximal dimension of the projected state on the center manifold associated with the generalized Bogdanov-Takens singularities from one side and $N$ the number of the delays and $n$ the degree of the polynomial $P_{0}$ from the other side. When $n_{0}$ the maximal degree of the polynomial family $\left(P_{i}\right)_{1 \leq i \leq N}$ is less than $n-1$ then a sharper upper bound for the dimension of the state in the center manifold is established.

More general systems with coupled delays even in the case of sparse polynomials will be considered in the future.

\section{APPENDIX}

We need first to introduce some notations. Let denote by $\Delta^{(k)}(\lambda)$ the $k$-th derivative of $\Delta(\lambda)$ with respect to the variable $\lambda$. We say that zero is an eigenvalue of algebraic multiplicity $m \geq 1$ for (1) if $\Delta(0)=\Delta^{(k)}(0)=0$ for all $k=1, \ldots, m-1$ and $\Delta^{(m)}(0) \neq 0$.

Let consider the non zero distinct delays such that $0<$ $\tau_{1}<\tau_{2}<\ldots<\tau_{N}$ and the polynomials $P_{i}$ such that $P_{0}$ is an unitary polynomial with $\operatorname{deg}\left(P_{0}\right)=n$ and $\operatorname{deg}\left(P_{i}\right) \leq$ $n-1$ for $1 \leq i \leq N$ and let $n_{0}=\max _{1 \leq i \leq N} \operatorname{deg}\left(P_{i}\right)$. We denote by $a_{i, k}$ the coefficient of the monomial $\lambda^{k}$ for the polynomial $P_{i}$, thus $a_{0, n}=1$.

Since we are dealing only with the values of $\Delta_{k}(0)$ we suggest to translate the problem into the parameter space (the space of the coefficients of the $P_{i}$ ), this will be more appropriate and will consider parametrization by $\tau$.

The following lemma allows to establish an $\mathrm{m}$-set of multivariate algebraic functions (polynomials) vanishing at zero when the multiplicity of the zero root of the transcendental equation $\Delta(\lambda, \tau)=0$ is equal to $m$.

Lemma 9: Zero is a root of $\Delta^{(k)}(\lambda)$ for $k \geq 0$ if and only if the coefficients of $P_{i}$ for $0 \leq i \leq N$ satisfy the following assertion

$$
a_{0, k}=-\sum_{i=1}^{N}\left[a_{i, k}-\sum_{l=0}^{k-1} \frac{(-1)^{l+k+1} a_{i, l} \tau_{i}^{k-l}}{(k-l) !}\right] .
$$

Let denote by

$$
\begin{aligned}
\nabla_{k}(\lambda) & =\sum_{i=0}^{N} \frac{d^{k}}{d \lambda^{k}} P_{i}(\lambda) \\
& +\sum_{l=0}^{k-1}\left((-1)^{l+k}\left(\begin{array}{l}
k \\
l
\end{array}\right) \sum_{i=1}^{N} \tau_{i}^{k-l} \frac{d^{l}}{d \lambda^{l}} P_{i}(\lambda)\right) .
\end{aligned}
$$

Now we are able to prove the main result:

Proof: [Proof of Proposition 1:] We shall consider the variety associated with the vanishing of the polynomials $\nabla_{k}$ (defined in lemma 9), that is $\nabla_{0}(0)=\ldots=\nabla_{m-1}(0)=0$ and $\nabla_{m}(0) \neq 0$ and we aim to find the maximal $m$ (codimension of the zero singularity). Consider the first elements from the family $\nabla_{k}$

$$
\begin{gathered}
\nabla_{0}(0)=0 \Leftrightarrow \sum_{i=0}^{N} a_{i, 0}=0, \\
\nabla_{1}(0)=0 \Leftrightarrow \sum_{i=0}^{N} a_{i, 1}-\sum_{i=1}^{N} a_{i, 0} \tau_{i}=0, \\
\nabla_{2}(0)=0 \Leftrightarrow 2 ! \sum_{i=0}^{N} a_{i, 1}-2 ! \sum_{i=1}^{N} a_{i, 0} \tau_{i}+\sum_{i=1}^{N} a_{i, 0} \tau_{i}^{2}=0,
\end{gathered}
$$


if we consider $a_{i, k}$ and $\tau_{l}$ as variables, the obtained algebraic system is nonlinear and solving it in all generality (without attributing values for $n$ and $N$ ) becomes a very difficult task. Indeed even by using Gröbner basis methods [23] this task is still complicated since the set of variables depends on $N, n$ and $n_{0}$. Since our aim is to establish an upper bound, we assume here that all the polynomials $P_{i}$ satisfy the condition $\operatorname{deg}\left(P_{i}\right)=n_{0}$ for all $1 \leq i \leq N$. We chose to consider $a_{i, k}$ as variables and $\tau_{l}$ as parameters and we adopt the following notation $a_{0}=\left(a_{0,0}, a_{0,1}, \ldots, a_{0, n-1}\right)^{T}$ and $a_{i}=\left(a_{i, 0}, a_{i, 1}, \ldots, a_{i, n_{0}}\right)^{T}$ for $1 \leq i \leq N$ and denote by $\tau=\left(\tau_{1}, \tau_{2}, \ldots, \tau_{N}\right)$ and $a=\left(a_{1}, a_{2}, \ldots, a_{N}\right)^{T}$. Consider first the ideal $I_{1}$ generated by the $n$ polynomials $<\nabla_{0}(0), \nabla_{1}(0), \ldots, \nabla_{n-1}(0)>$. As it can be seen from lemma 9, the variety $V_{1}$ associated with the ideal $I_{1}$ has the following linear representation $a_{0}=M_{1}(\tau) a$ where $M_{1} \in \mathcal{M}_{n, N\left(n_{0}+1\right)}(\mathbb{R}[\tau])$. In some sense, in this variety there are no any restriction on the components of $a$ when $a_{0}$ is left free. Since $a_{0, k}=0$ for all $k>n$, the remaining equations consist of an algebraic system only in $a$ and parametrized by $\tau$. Consider now the ideal denoted $I_{2}$ and generated by the $N\left(n_{0}+1\right)$ polynomials defined by

$$
I_{2}=<\nabla_{n+1}(0), \nabla_{n+2}(0), \ldots, \nabla_{n+N\left(n_{0}+1\right)}(0)>.
$$

It can be observed that the variety $V_{2}$ associated with $I_{2}$ can be written as $M_{2}(\tau) a=0$ which is nothing else that an homogeneous linear system with $M_{2} \in \mathcal{M}_{N\left(n_{0}+1\right)}(\mathbb{R}[\tau])$. More precisely, $M_{2}$ is a generalized Vandermonde matrice

$$
\begin{aligned}
M_{2}(\tau)= & \left(V\left(\tau_{1}\right), \frac{d}{d \tau_{1}} V\left(\tau_{1}\right), \ldots, \frac{d^{n_{0}}}{d \tau_{1}^{n_{0}}} V\left(\tau_{1}\right), \ldots, V\left(\tau_{N}\right),\right. \\
& \left.\ldots, \frac{d^{n_{0}}}{d \tau_{N}^{n_{0}}} V\left(\tau_{N}\right)\right), \text { where } \\
V(x)= & \left((-x)^{n+1},(-x)^{n+2}, \ldots,(-x)^{n+N\left(n_{0}+1\right)}\right)^{T}
\end{aligned}
$$

Obviously, every subset of vectors $F_{k}=$ $\left(V\left(\tau_{k}\right), \ldots, \quad \frac{d^{n_{0}}}{d \tau_{k}^{n_{0}}} V\left(\tau_{k}\right)\right)$ is a family of vectors in $\mathbb{R}^{N(n-1)}\left(\left[\tau_{k}\right]\right)$, which are linearly independent since, as it can be seen in (A.2) that, for any $i \neq l, \operatorname{deg}\left(V_{\mathrm{i}}\right) \neq \operatorname{deg}\left(\mathrm{V}_{\mathrm{l}}\right)$, where $V_{k}$ is the $k$-th component of the vector $V$ and $\operatorname{deg}\left(\mathrm{V}_{\mathrm{k}}\right)$ denotes the degree of the polynomial $V_{k}(x)$ in $x$. Moreover, no any element from $F_{l}$ (the family of vectors in $\mathbb{R}^{N(n-1)}\left(\left[\tau_{l}\right]\right)$ ) can be written as a linear combination of elements of $F_{k}$ with $l \neq k$, which proves that $\operatorname{det}(\mathrm{M})$ can not vanish. Furthermore, the direct computation of the determinant of the matrix $M$ gives

$$
\begin{aligned}
&|\operatorname{det}(M)|=\mid \prod_{1 \leq k \leq n-2}\left(n_{0}+1-k\right) !^{N} \mid \times \\
&\left|\prod_{1 \leq i<l \leq N}\left(\tau_{i}-\tau_{l}\right)^{\left(n_{0}+1\right)^{2}} \prod_{1 \leq h \leq N} \tau_{h}^{\left(n_{0}+1\right)(n+1)}\right| .
\end{aligned}
$$

Since we are concerned only by non zero distinct delays, then this determinant can not vanish. Thus the only solution for this subsystem is the zero solution, that is $a=0$.
Now consider the polynomial defined by $\nabla_{n}(0)$, by lemma 9 (see appendix)

$$
\nabla_{n}(0)=0 \Leftrightarrow 1=\sum_{i=1}^{N} \sum_{l=0}^{n-1} \frac{(-1)^{l+n+1} a_{i, l} \tau_{i}^{n-l}}{(n-l) !}
$$

substituting the unique solution of $V_{2}$ into the last equality leads to an incompatibility result. In conclusion, the maximal codimension of the zero singularity is less or equal to $N\left(n_{0}+\right.$ 1) $+n$.

\section{REFERENCES}

[1] L. V. Ahlfors, Complex Analysis, McGraw-Hill, Inc., 1979.

[2] R. Bellman, K. L. Cooke, Differential-difference equations, Academic Press, New York, 1963.

[3] W. Michiels, S.-I. Niculescu, Stability and stabilization of time-delay systems, Vol. 12 of Advances in Design and Control, Society for Industrial and Applied Mathematics (SIAM), Philadelphia, PA, 2007, an eigenvalue-based approach.

[4] J. K. Hale, W. Huang, Period doubling in singularly perturbed delay equations, Journal of Differential Equations 114 (1994) 1-23.

[5] I. Boussaada, H. Mounier, S.-I. Niculescu, A. Cela, Control of drilling vibrations: A time-delay system approach, MED 2012, 20th Mediterranean Conference on Control and Automation, Barcelona (2012) 5pp.

[6] S. Campbell, Y. Yuan, Zero singularities of codimension two and three in delay differential equations, Nonlinearity 21 (11) (2008) 2671.

[7] J. Sieber, B. Krauskopf, Bifurcation analysis of an inverted pendulum with delayed feedback control near a triple-zero eigenvalue singularity, Nonlinearity 17 (2004) 85-103.

[8] I. Boussaada, I.-C. Morarescu, S.-I. Niculescu, Inverted pendulum stabilization: Characterisation of codimension-three triple zero bifurcation via multiple delayed proportional gains, Preprint (2012) 1-21.

[9] J. Carr, Application of Center Manifold Theory, Springer, 1981.

[10] J. K. Hale, S. M. V. Lunel, Introduction to functional differential equations, Vol. 99 of Applied Mathematics Sciences, Springer Verlag, New York, 1993.

[11] J. Guckenheimer, P. Holmes, Nonlinear oscillations, dynamical systems, and bifurcation of vector fields, Springer, 2002.

[12] Y. Kuznetsov, Elements of applied bifurcation theory; Second edition, Vol. 112 of Applied Mathematics Sciences, Springer, New York, 1998.

[13] T. Kailath, Linear Systems, Prentice-Hall information and system sciences series, Prentice Hall International, 1998.

[14] S.-I. Niculescu, W. Michiels, Stabilizing a chain of integrators using multiple delays, IEEE Trans. on Aut. Cont. 49 (5) (2004) 802-807.

[15] F. M. Atay, Balancing the inverted pendulum using position feedback, Appl. Math. Lett. 12 (5) (1999) 51-56.

[16] J. Sieber, B. Krauskopf, Extending the permissible control loop latency for the controlled inverted pendulum, Dynamical Systems 20 (2) (2005) 189-199.

[17] M. Giesbrecht, D.-S. Roche, Interpolation of shifted-lacunary polynomials, Computational Complexity (2010) 1-21.

[18] M. Marden, Geometry of Polynomials, Mathematical Surveys 3, Providence, AMS, 1966.

[19] G. Polya, G. Szegö, Problems and Theorems in Analysis, Vol. I: Series, Integral Calculus, Theory of Functions, Springer-Verlag, New York, Heidelberg, and Berlin, 1972.

[20] I. Boussaada, S.-I. Niculescu, Computing the codimension of the singularity at the origin for delay systems: The missing link with birkhoff incidence matrices, The 21th International Symposium on Mathematical Theory of Networks and Systems (MTNS) (2014) 1-8.

[21] K. Furuta, T. Okutani, H. Sone, Computer control of a double inverted pendulum, Computers \& Electrical Engineering 5 (1) (1978) 67 - 84.

[22] A. Bogdanov, Optimal control of a double inverted pendulum on a cart, Tech. rep., CSEE, OGI School of Science and Engineering, OHSU (2004).

[23] D. Cox, J. Little, D. O'Shea, Ideals, varieties, and algorithms. An introduction to computational algebraic geometry and commutative algebra, Undergraduate Texts in Mathematics, Springer, New York, 2007. 